\title{
Spin quantum tunneling via entangled states in a dimer of exchange coupled single-molecule magnets
}

\author{
R. Tiron ${ }^{1}$, W. Wernsdorfer ${ }^{1}$, D. Foguet-Albiol ${ }^{2}$, N. Aliaga-Alcalde ${ }^{2}$, G. Christou ${ }^{2}$ \\ ${ }^{1}$ Lab. L. Néel, associé à l'UJF, CNRS, BP 166, 38042 Grenoble Cedex 9, France \\ ${ }^{2}$ Dept. of Chemistry, Univ. of Florida, Gainesville, Florida 32611-7200, USA
}

(Dated: 18th July 2003, submitted to PRL)

\begin{abstract}
A new family of supramolecular, antiferromagnetically exchange-coupled dimers of single-molecule magnets (SMMs) has recently been reported [W. Wernsdorfer, N. Aliaga-Alcalde, D.N. Hendrickson, and G. Christou, Nature 416, 406 (2002)]. Each SMM acts as a bias on its neighbor, shifting the quantum tunneling resonances of the individual SMMs. Hysteresis loop measurements on a single crystal of SMM-dimers have now established quantum tunneling of the magnetization via entangled states of the dimer. This shows that the dimer really does behave as a quantum-mechanically coupled dimer. The transitions are well separated, suggesting long coherence times compared to the time scale of the energy splitting. This result is of great importance if such systems are to be used for quantum computing. It also allows the measurement of the longitudinal and transverse superexchange coupling constants.
\end{abstract}

PACS numbers: $75.45 .+\mathrm{j}, 75.60 . \mathrm{Ej}, 75.50 . \mathrm{Xx}$

Single-molecule magnets (SMM) are among the smallest nanomagnets that exhibit magnetization hysteresis, a classical property of macroscopic magnets [1, 2, 3, 4, [5]. They straddle the interface between classical and quantum mechanical behavior because they also display quantum tunneling of magnetization $6,7,8,9,10,11$, 12, 13 and quantum phase interference 14, 15. These molecules comprise several magnetic ions, whose spins are coupled by strong exchange interactions to give a large effective spin. The molecules are regularly assembled within large crystals, with all the molecules often having the same orientation. Hence, macroscopic measurements can give direct access to single molecule properties. Many non-magnetic atoms surround the magnetic core of each molecule. Exchange interactions between molecules are therefore relatively weak and have been neglected in most studies. Recently, the study of a dimerized SMM, $\left[\mathrm{Mn}_{4}\right]_{2}$, showed that intermolecular exchange interactions are not always negligible and can instead be used to couple SMMs. This system [16] was $\left[\mathrm{Mn}_{4} \mathrm{O}_{3} \mathrm{Cl}_{4}\left(\mathrm{O}_{2} \mathrm{CEt}\right)_{3}(\mathrm{py})_{3}\right]_{2}$ (hereafter called $\left.\left[\mathrm{Mn}_{4}\right]_{2}\right)$, a member of the $\left[\mathrm{Mn}_{4} \mathrm{O}_{3} \mathrm{Cl}_{4}\left(\mathrm{O}_{2} \mathrm{CR}\right)_{3}(\mathrm{py})_{3}\right]_{2}$ family, with $\mathbf{R}=$ Et . The crystal form studied, $\left[\mathrm{Mn}_{4}\right]_{2} \cdot 8 \mathrm{MeCN}$, was that obtained from MeCN solution, containing $4 \mathrm{MeCN}$ solvent molecules of crystallization per $\mathrm{Mn}_{4}$. The spins of the two $\mathrm{Mn}_{4}$ molecules are coupled antiferromagnetically. Each molecule acts as a bias on its neighbor, the quantum tunneling resonances thus being shifted with respect to the isolated $\mathrm{Mn}_{4} \mathrm{SMM}$. The first three-dimensional networks of exchange coupled SMMs have also been studied recently [17, 18].

In this letter, we present new results discovered on a different crystal form of the same $\left[\mathrm{Mn}_{4}\right]_{2}$ compound, obtained from $\mathrm{CH}_{2} \mathrm{Cl}_{2} / \mathrm{Et}_{2} \mathrm{O} / \mathrm{C}_{6} \mathrm{H}_{14}$ solution and containing one hexane $\left(\mathrm{C}_{6} \mathrm{H}_{14}\right)$ molecule of crystallization per $\mathrm{Mn}_{4}$ i.e. $\left[\mathrm{Mn}_{4}\right]_{2} \cdot 2 \mathrm{C}_{6} \mathrm{H}_{14}$. Both $\left[\mathrm{Mn}_{4}\right]_{2} \cdot 8 \mathrm{MeCN}$ and
$\left[\mathrm{Mn}_{4}\right]_{2} \cdot 2 \mathrm{C}_{6} \mathrm{H}_{14}$ crystallize isomorphously, but the latter has a stronger intradimer and negligible interdimer exchange interactions compared with the former, and was thus better suited for the studies presented here. We have identified for the first time quantum tunneling transitions via entangled states of the $\left[\mathrm{Mn}_{4}\right]_{2}$ dimer. The corresponding energy levels are well separated, showing that the decoherence in this system is small. In our previous report, we did not have, and thus could not provide, evidence for quantum mechanical entanglement within this dimer, but the present results establish that the dimer really does behave as a quantum-mechanically coupled system.

The compound $\left[\mathrm{Mn}_{4}\right]_{2} \cdot 2 \mathrm{C}_{6} \mathrm{H}_{14}$ crystallizes in the hexagonal space group $R 3$ (bar) with two $\mathrm{Mn}_{4}$ molecules per unit cell lying head-to-head on a crystallographic $\mathrm{S}_{6}$ symmetry axis 20, as does previously reported $\left[\mathrm{Mn}_{4}\right]_{2} \cdot 8 \mathrm{MeCN}[16]$. Each $\mathrm{Mn}_{4}$ monomer has a ground state spin of $S=9 / 2$, well separated from the first excited state $S=7 / 2$ by a gap of about $300 \mathrm{~K}$ 19]. The $\mathrm{Mn}-\mathrm{Mn}$ distances and the Mn-O-Mn angles are similar and the uniaxial anisotropy constant is expected to be the same for the two dimer systems. These dimers are held together via six $\mathrm{C}-\mathrm{H} \cdots \mathrm{Cl}$ hydrogen bonds between the pyridine (py) rings on one molecule and the $\mathrm{Cl}$ ions on the other, and one $\mathrm{Cl}$. Cl Van der Waals interaction. These interactions lead to an antiferromagnetic superexchange interaction between the two $\mathrm{Mn}_{4}$ units of the $\left[\mathrm{Mn}_{4}\right]_{2}$ dimer 16]. Dipolar couplings between $\mathrm{Mn}_{4}$ molecules can be easily calculated and are more than one order of magnitude smaller than the exchange interaction.

Before presenting the measurements, we summarize a simplified spin Hamiltonian describing the $\left[\mathrm{Mn}_{4}\right]_{2}$ dimer [16]. Each $\mathrm{Mn}_{4} \mathrm{SMM}$ can be modeled as a giant spin of $S=9 / 2$ with Ising-like anisotropy. The corre- 


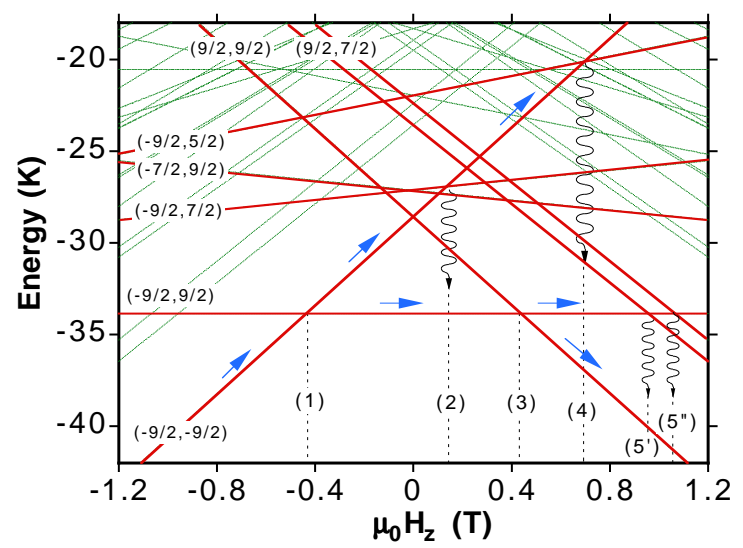

FIG. 1: (Online color) Low lying spin state energies of the $\left[\mathrm{Mn}_{4}\right]_{2}$ dimer, calculated by exact numerical diagonalization using Eq. 2 with $D=0.77 \mathrm{~K}$ and $J=0.13 \mathrm{~K}$, as a function of applied magnetic field $H_{z}$ (Zeeman diagram). The bold energy levels are labelled with two quantum numbers $\left(M_{1}, M_{2}\right)$. Dotted lines, labelled $\mathbf{1}$ to $\mathbf{5}$, indicate the strongest tunnel resonances: 1: $(-9 / 2,-9 / 2)$ to $(-9 / 2,9 / 2) ; \mathbf{2}:(-9 / 2,-9 / 2)$ to $(-$ $9 / 2,7 / 2)$, followed by relaxation to $(-9 / 2,9 / 2) ; \mathbf{3}:(-9 / 2,9 / 2)$ to $(9 / 2,9 / 2) ; 4:(-9 / 2,-9 / 2)$ to $(-9 / 2,5 / 2)$, followed by relaxation to $(-9 / 2,9 / 2) ; \mathbf{5}:(-9 / 2,9 / 2)$ to $(7 / 2,9 / 2)$, followed by relaxation to $(9 / 2,9 / 2)$. For clarity, degenerate states such as (M,M') and (M',M) and lifted degenerate states such as $(M, M \pm 1),(M, M \pm 2) \ldots$ are not both listed. For example, the $(9 / 2,7 / 2)$ and $(7 / 2,9 / 2)$ states are strongly split into a symmetric (labelled $5^{\prime \prime}$ ) and antisymmetric (labelled $5^{\prime}$ ) combination of $(9 / 2,7 / 2)$ and $(7 / 2,9 / 2)$ states. This splitting is used to measure the transverse superexchange interaction constant $J_{x y}$. Co-tunneling and other two-body tunnel transitions have a lower probability of occurrence and are neglected [21].

sponding Hamiltonian is given by

$$
\mathcal{H}_{i}=-D S_{z, i}^{2}+\mathcal{H}_{\text {trans }, i}+g \mu_{\mathrm{B}} \mu_{0} \vec{S}_{i} \cdot \vec{H}
$$

where $i=1$ or 2 (referring to the two $\mathrm{Mn}_{4}$ SMMs of the dimer), $D$ is the uniaxial anisotropy constant, and the other symbols have their usual meaning. Tunneling is allowed in these half-integer $(S=9 / 2)$ spin systems because of a small transverse anisotropy $\mathcal{H}_{\text {trans }, i}$ containing $S_{x, i}$ and $S_{y, i}$ spin operators and transverse fields $\left(H_{x}\right.$ and $\left.H_{y}\right)$. The exact form of $\mathcal{H}_{\text {trans }, i}$ is not important in this discussion. The last term in Eq. 1 is the Zeeman energy associated with an applied field. The $\mathrm{Mn}_{4}$ units within the $\left[\mathrm{Mn}_{4}\right]_{2}$ dimer are coupled by a weak superexchange interaction via both the six $\mathrm{C}-\mathrm{H} \cdots \mathrm{Cl}$ pathways and the $\mathrm{Cl} \cdots \mathrm{Cl}$ approach. Thus, the Hamiltonian $(\mathcal{H})$ for $\left[\mathrm{Mn}_{4}\right]_{2}$ is

$$
\mathcal{H}=\mathcal{H}_{1}+\mathcal{H}_{2}+J_{z} S_{z, 1} S_{z, 2}+J_{x y}\left(S_{x, 1} S_{x, 2}+S_{y, 1} S_{y, 2}\right)
$$

where $J_{z}$ and $J_{x y}$ are respectively the longitudinal and transverse superexchange interactions. $J_{z}=J_{x y}$ is the case of isotropic superexchange. The $(2 S+1)^{2}=100$

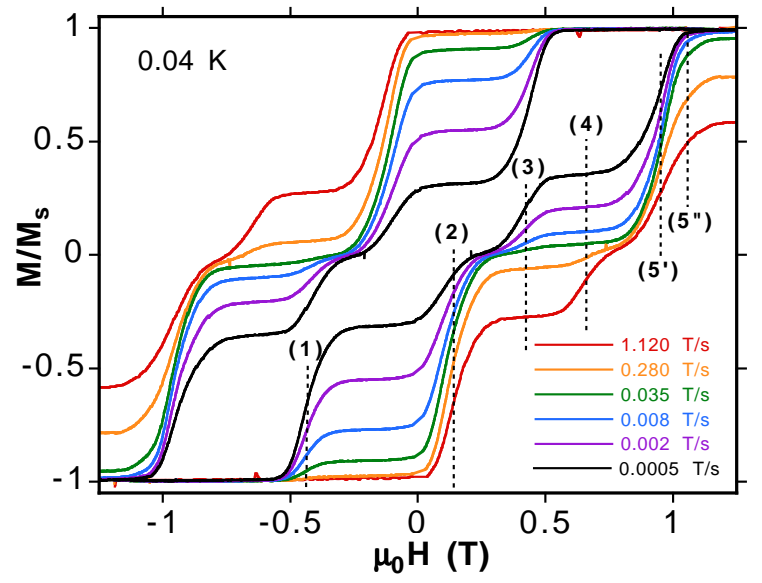

FIG. 2: (Online color) Hysteresis loops for the $\left[\mathrm{Mn}_{4}\right]_{2}$ dimer at several field sweep rates and $40 \mathrm{mK}$. The tunnel transitions (manifested by steps) are labelled from $\mathbf{1}$ to $\mathbf{5}$, see Fig. 1.

energy states of the dimer can be calculated by exact numerical diagonalization and are plotted in Fig. 1 as a function of applied field along the easy axis. Each state of $\left[\mathrm{Mn}_{4}\right]_{2}$ can be labelled by two quantum numbers $\left(M_{1}, M_{2}\right)$ for the two $\mathrm{Mn}_{4} \mathrm{SMMs}$, with $M_{1}=-9 / 2,-7 / 2, \ldots, 9 / 2$ and $M_{2}=-9 / 2,-7 / 2, \ldots, 9 / 2$. The degeneracy of some of the $\left(M_{1}, M_{2}\right)$ states is lifted by transverse anisotropy terms. For the sake of simplicity, we will discuss mainly the effect of the transverse superexchange interaction $\mathcal{J}_{\text {trans }}=J_{x y}\left(S_{x, 1} S_{x, 2}+\right.$ $\left.S_{y, 1} S_{y, 2}\right)=J_{x y}\left(S_{+, 1} S_{-, 2}+S_{-, 1} S_{+, 2}\right) / 2$, where $S_{+, i}$ and $S_{-, i}$ are the usual spin raising and lowering operators. Because $\mathcal{J}_{\text {trans }}$ acts on $(M, M \pm 1)$ states to first order of perturbation theory, the degeneracy of those states is strongly lifted. For example, the $(9 / 2,7 / 2)$ and $(7 / 2,9 / 2)$ states are strongly split into a symmetric (labelled $5^{\prime \prime}$ ) and antisymmetric (labelled $\left.5^{\prime}\right)$ combination of $(9 / 2,7 / 2)$ and $(7 / 2,9 / 2)$ states. Similarly for the $(-9 / 2,-7 / 2)$ and ($7 / 2,-9 / 2)$ states. Measuring this energy splitting allows us to determine the transverse superexchange interaction constant $J_{x y}$ because the latter is proportional to the former.

Tunneling studies on $\left[\mathrm{Mn}_{4}\right]_{2}$ were performed by magnetization measurements on single crystals using an array of micro-SQUIDs 22]. Fig. 2 shows typical hysteresis loops (magnetization versus magnetic field scans) with the field applied along the easy axis of magnetization of $\left[\mathrm{Mn}_{4}\right]_{2}$, that is, parallel to the $\mathrm{S}_{6}$ axis. These loops display step-like features separated by plateaus. The step heights are temperature-independent below $\sim 0.35 \mathrm{~K}$ (not shown). The steps are due to resonant quantum tunneling of the magnetization (QTM) between the energy states of the $\left[\mathrm{Mn}_{4}\right]_{2}$ dimer (see figure caption 1 and 2 for a discussion of 5 tunnel transitions). QTM has been previously observed for most SMMs, but the novelty for 
$\left[\mathrm{Mn}_{4}\right]_{2}$ dimers is that the QTM is now the collective behavior of the complete $S=0$ dimer of exchange-coupled $S=9 / 2 \mathrm{Mn}_{4}$ quantum systems. This coupling is manifested as an exchange bias of all tunneling transitions, and the resulting hysteresis loop consequently displays unique features, such as the absence for the first time in a SMM of a QTM step at zero field [16.

Even though the five strongest tunneling transitions are observed in Fig. 2, fine structure was not observed. For example, the hysteresis loops do not show the splitting of the $(9 / 2,7 / 2)$ states (labelled $5^{\prime}$ and $\left.5^{\prime \prime}\right)$, which we suspected might be due to line broadening. Usually, line broadening in SMMs is caused by dipolar and hyperfine interactions [23], and distributions of anisotropy and exchange parameters. In most SMMs, the zero-field resonance is mainly broadened by dipolar and hyperfine interactions because distributions of anisotropy parameters do not affect the zero-field resonance. For an antiferromagnetically coupled dimer, however, this resonance is shifted to negative fields. Therefore, a distribution of the exchange coupling parameter $J_{z}$ can further broaden this resonance. In fact, we show in the following that the latter is the dominant source of broadening. We then use the 'quantum hole-digging' method [23, 24, 25, 26, 27] to provide direct experimental evidence for the transitions $5^{\prime}$ and $5^{\prime \prime}$, which establishes tunneling involving entangled dimer states and allows us to determine $J_{x y}$.

The 'quantum hole-digging' method is a relatively new method that can, among other things 13], study line broadening and its evolution during relaxation [23, 24, 25, 26, 27]. The method is based on the simple idea that after a rapid field change, the resulting magnetization relaxation at short time periods is directly related to the number of molecules in resonance at the applied field; Prokof'ev and Stamp proposed [23] that this short time relaxation should follow a $\sqrt{t}(t=$ time) relaxation law. Thus, the magnetization of the $\left[\mathrm{Mn}_{4}\right]_{2}$ dimers in the crystal was first saturated with a large positive field, and then a 'digging field' $H_{\text {dig }}$ was applied at $0.04 \mathrm{~K}$ for a chosen 'digging time' $t_{\mathrm{dig}}$. Then, the fraction (and only that fraction) of the molecules that is in resonance at $H_{\text {dig }}$ can undergo magnetization tunneling. After $t_{\text {dig }}$, a field $H_{\text {probe }}$ is applied and the magnetization relaxation rate is measured for short time periods; from this is calculated the short-time relaxation rate $\Gamma_{\text {sqrt }}$, which is related to the number of $\left[\mathrm{Mn}_{4}\right]_{2}$ dimers still available for QTM 22]. The entire procedure is then repeated at other $H_{\text {probe }}$ fields. The resulting plot (Fig. 3a) of $\Gamma_{\text {sqrt }}$ versus $H_{\text {probe }}$ reflects the distribution of spins still available for tunneling after $t_{\text {dig. }}$.

In the limit of very short digging times, the difference between the relaxation rate in the absence and in the presence of digging, $\Gamma_{\text {hole }}=\Gamma_{\text {sqrt }}\left(t_{\text {dig }}=0\right)-\Gamma_{\text {sqrt }}\left(t_{\text {dig }}\right)$, is approximately proportional to the number of molecules which reversed their magnetization during the time $t_{\text {dig }}$ (Fig. $3 b) . \Gamma_{\text {hole }}$ is characterized by a width that can be

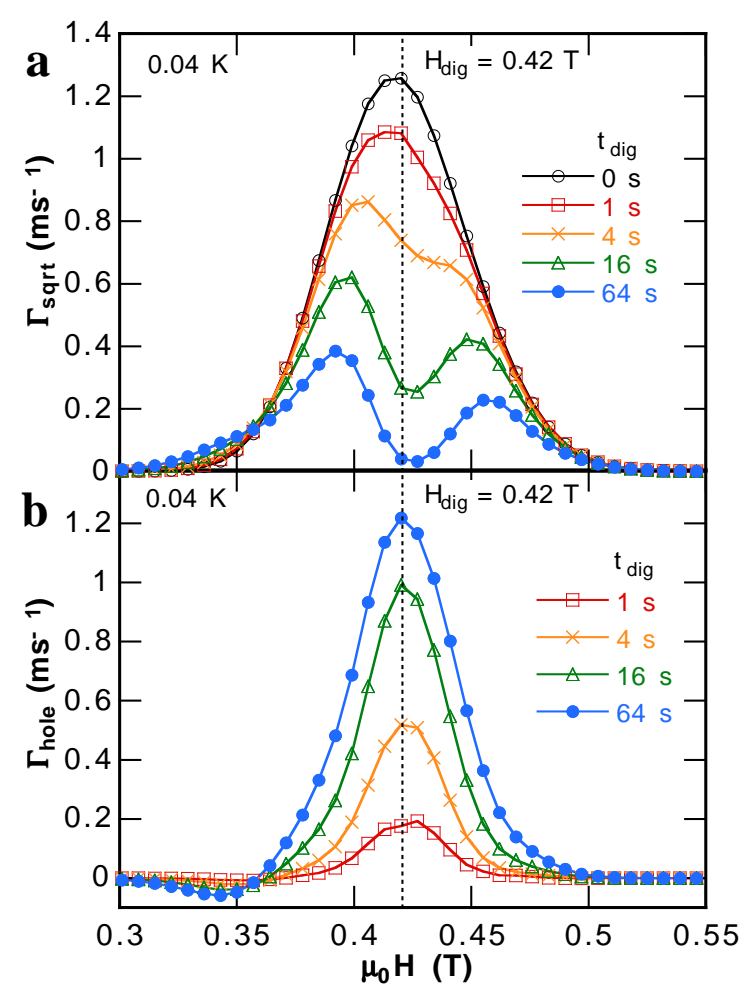

FIG. 3: (Online color) (a) Field dependence of the shorttime square-root relaxation rates $\Gamma_{\text {sqrt }}$ are presented on a logarithmic scale showing the depletion of the molecular spin states by quantum tunneling at $H_{\mathrm{dig}}=0.42 \mathrm{~T}$ for various waiting times $t_{\text {dig. }}$. (b) Difference between the relaxation rate in the absence and in the presence of digging, $\Gamma_{\text {hole }}=\Gamma_{\text {sqrt }}\left(t_{\text {dig }}=0\right)-\Gamma_{\text {sqrt }}\left(t_{\text {dig }}\right)$.

called the 'tunnel window'.

The width of the distribution in the absence of digging ( $\sim 80 \mathrm{mT}$, Fig. 3a) is too large to be due to only dipolar $(\sim 20 \mathrm{mT})$ and hyperfine coupling $(\sim 10 \mathrm{mT})$. The following result suggests that it is due to a distribution of the exchange coupling parameter $J_{z}$.

First, the magnetization of the $\left[\mathrm{Mn}_{4}\right]_{2}$ dimers was saturated with a large positive field, and then a 'digging field' $H_{\text {dig }}$ was applied to reverse a fraction of the molecules that are in resonance at $H_{\text {dig }}$ (transition 3 in Fig. 1). After the reversal of $2.5 \%$ of the molecules, the applied field is swept back to a large positive field. $5^{\prime}$ and $5^{\prime \prime}$ are the first tunnel transitions that can allow the reversed molecules to tunnel back to positive saturation. Figs. 4a and $4 \mathrm{~b}$ show the corresponding minor hysteresis loops for several 'digging fields and field sweep rates, respectively. Both figures show clearly the expected tunnel transitions $5^{\prime}$ and $5^{\prime \prime}$, that were not resolved in the major hysteresis loops (Fig. 2). This suggests that the broadening of tunneling transition 3 (the distribution in the absence of digging in Fig. 3a) is dominated by a distribution of the 


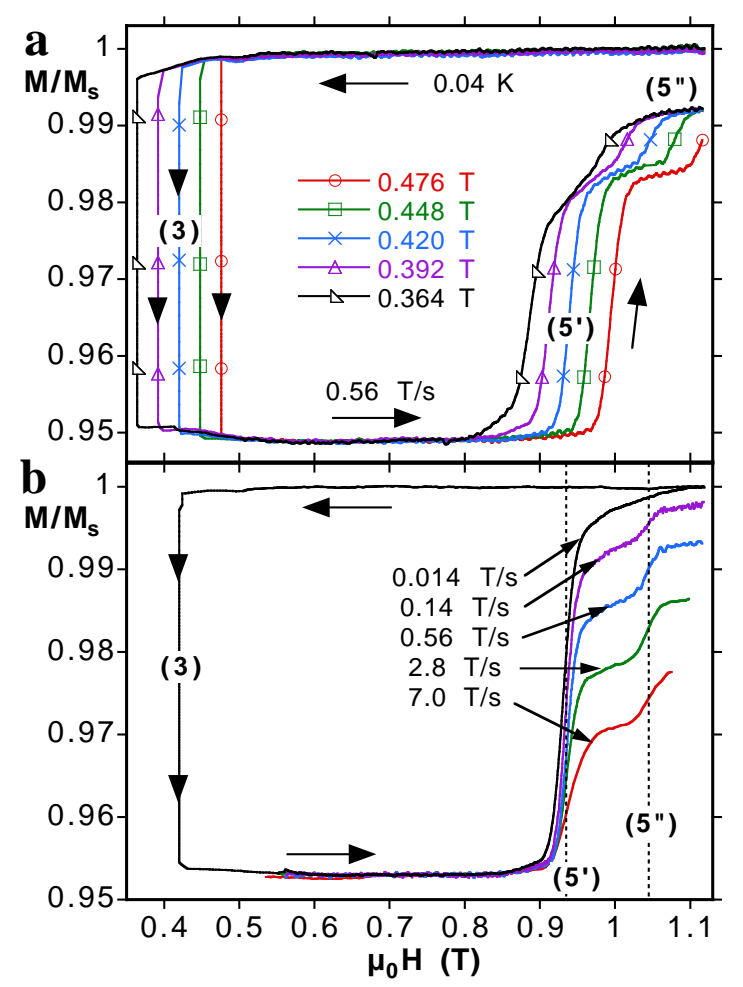

FIG. 4: (Online color) Minor hysteresis loops for several (a) digging fields and (b) field sweep rates. After positive saturation, a digging field $H_{\text {dig }}$ was applied to reverse $\approx 2.5 \%$ of the molecules that are in resonance at $H_{\text {dig }}$ (transition $\mathbf{3}$ in Fig. $1)$. Then, the applied field is swept back to a large positive field. $5^{\prime}$ and $5^{\prime \prime}$ are the first tunnel transitions allowing the reversed molecules to tunnel back to positive saturation.

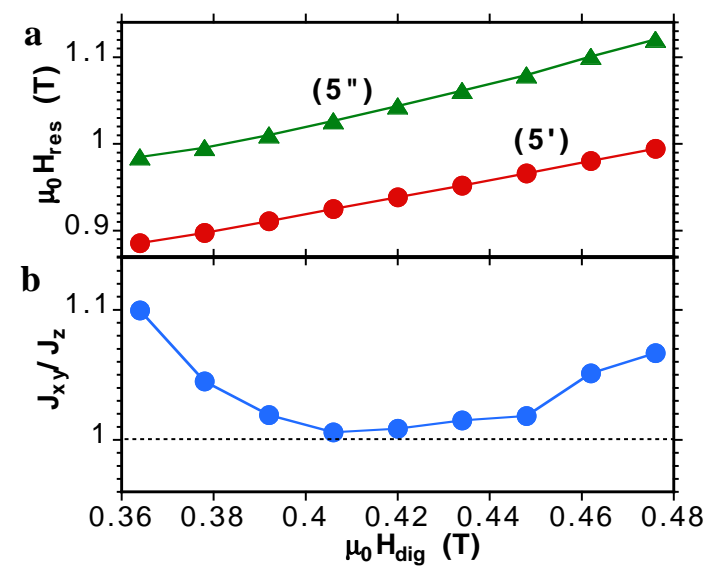

FIG. 5: (Online color) (a) Resonance field positions $H_{\text {res }}$ of $5^{\prime}$ and $5^{\prime \prime}$ and (b) normalized transverse superexchange interaction $J_{x y} / J_{z}$ as a function of digging field $H_{\mathrm{dig}}$. exchange coupling parameter $J_{z}$. During the application of the digging field, a subgroup of molecules is selected with an exchange coupling constant $J_{z} \approx g \mu_{\mathrm{B}} \mu_{0} H_{\mathrm{dig}} / S$, that can tunnel back at the fields of transitions $5^{\prime}$ and $5^{\prime \prime}$.

This interpretation is supported by the study of the field values of $5^{\prime}$ and $5^{\prime \prime}$ as a function of digging field, that is $J_{z}$, exhibiting a nearly linear variation (Fig. 5a). The field difference between transition $5^{\prime}$ and $5^{\prime \prime}$ can be used to find the $J_{x y}$, presented in Fig. 5b. This shows that the superexchange interaction of the dimers is nearly isotropic $\left(J_{x y} \approx J_{z}\right)$. It is important to mention that the transitions $5^{\prime}$ and $5^{\prime \prime}$ are well separated, suggesting long coherence times compared to the time scale of the energy splitting.

The above results demonstrate for the first time tunneling via entangled states of a dimer of exchange coupled SMMs, showing that the dimer really does behave as a quantum mechanically coupled system. This result is of great importance if such systems are to be used for quantum computing.

We thank the CNRS, Rhone-Alpe, and USA National Science Foundation for support.

[1] G. Christou, D. Gatteschi, D.N. Hendrickson, and R. Sessoli, MRS Bulletin 25, 66 (2000).

[2] R. Sessoli, H.-L. Tsai, A. R. Schake, S. Wang, J. B. Vincent, K. Folting, D. Gatteschi, G. Christou, and D. N. Hendrickson, J. Am. Chem. Soc. 115, 1804 (1993).

[3] R. Sessoli, D. Gatteschi, A. Caneschi, and M. A. Novak, Nature 365, 141 (1993).

[4] S. M. J. Aubin and et al., J. Am. Chem. Soc. 118, 7746 (1996).

[5] C. Boskovic and et al., J. Am. Chem. Soc. 124, 3725 (2002).

[6] M.A. Novak and R. Sessoli, in Quantum Tunneling of Magnetization-QTM'94, Vol. 301 of NATO ASI Series E: Applied Sciences, edited by L. Gunther and B. Barbara (Kluwer Academic Publishers, London, 1995), pp. 171188.

[7] J. R. Friedman, M. P. Sarachik, J. Tejada, and R. Ziolo, Phys. Rev. Lett. 76, 3830 (1996).

[8] L. Thomas, F. Lionti, R. Ballou, D. Gatteschi, R. Sessoli, and B. Barbara, Nature (London) 383, 145 (1996).

[9] C. Sangregorio, T. Ohm, C. Paulsen, R. Sessoli, and D. Gatteschi, Phys. Rev. Lett. 78, 4645 (1997).

[10] S. Hill and et al., Phys. Rev. Lett. 80, 2453 (1998).

[11] S. M. J. Aubin, N. R. Dilley, M. B. Wemple, G. Christou, and D. N. Hendrickson, J. Am. Chem. Soc. 120, 839 (1998).

[12] L. Bokacheva, A.D. Kent, and M.A. Walters, Phys. Rev. Lett. 85, 4803 (2000).

[13] W. Wernsdorfer, M. Soler, D. N. Hendrickson, and G. Christou, cond-mat/0306303

[14] W.Wernsdorfer and R. Sessoli, Science 284, 133 (1999).

[15] A. Garg, EuroPhys. Lett. 22, 205 (1993).

[16] W. Wernsdorfer, N. Aliaga-Alcalde, D.N. Hendrickson, 
and G. Christou, Nature 416, 406 (2002).

[17] E. Yang, W. Wernsdorfer, S. Hill, R.S. Edwards, M. Nakano, S. Maccagnano, L.N. Zakharov, A. L. Rheingold, G. Christou, and D.N. Hendrickson, Polyhedron 22, 1727 (2003).

[18] R. Tiron, W. Wernsdorfer, N. Aliaga-Alcalde, D.N. Hendrickson, and G. Christou, cond-mat/0306669

[19] D. N. Hendrickson and et al., J. Am. Chem. Soc. 114, 2455 (1992).

[20] N. Aliaga-Alcalde and et al., manuscript in preparation.

[21] W. Wernsdorfer, S. Bhaduri, R. Tiron, D.N. Hendrickson, G. Christou, Phys. Rev. Lett. 89 (2002) 197201.
[22] W. Wernsdorfer, Adv. Chem. Phys. 118, 99 (2001).

[23] N.V. Prokof'ev and P.C.E. Stamp, Phys. Rev. Lett. 80, 5794 (1998).

[24] W. Wernsdorfer, T. Ohm, C. Sangregorio, R. Sessoli, D. Mailly, and C. Paulsen, Phys. Rev. Lett. 82, 3903 (1999).

[25] W. Wernsdorfer, A. Caneschi, R. Sessoli, D. Gatteschi, A. Cornia, V. Villar, and C. Paulsen, Phys. Rev. Lett. 84, 2965 (2000).

[26] J. J. Alonso and J. F. Fernandez, Phys. Rev. Lett. 87, 097205 (2001).

[27] I. Tupitsyn and P.C.E. Stamp, cond-mat/0305371 